\title{
Effect of Farm Saved Maize (Zea mays L.) Seed on Intensity of Foliage Diseases
}

\author{
Alfred N. Mutundi ${ }^{1,2}$, James W. Muthomi ${ }^{1}$, Florence M. Olubayo ${ }^{1}$, Philip K. Leley ${ }^{3}$ \& Felista M. Nzuve ${ }^{1}$ \\ ${ }^{1}$ Department of Plant Science and Crop Protection, University of Nairobi, Nairobi, Kenya \\ ${ }^{2}$ National Seeds Certification, North-Kivu, Democratic Republic of Congo \\ ${ }^{3}$ Kenya Agricultural and Livestock Research Organization, Kenya \\ Correspondence: Alfred N. Mutundi, National Seeds Certification, 262 Av. Kahembe, Q/Bahizi Airport Route, \\ Karisimbi-Goma Town, North-Kivu, Democratic Republic of Congo. E-mail: alfredmutundi@gmai.com
}

Received: March 2, 2019

doi:10.5539/jas.v11n8p45
Accepted: April 19, $2019 \quad$ Online Published: June 15, 2019

URL: https://doi.org/10.5539/jas.v11n8p45

\begin{abstract}
Majority of farmers in Africa recycle farm saved seed from the previous seasons. Such seed is usually contaminated with disease causing pathogens, has low vigor and result in low yields. This study was, therefore, conducted to determine the effect of recycling maize seeds on incidence of foliage diseases. Maize seeds were collected during 2016 short rain season from farmers, local market and Agrovet shops in Busia County of western Kenya. The seeds were subjected to field evaluation during 2017 long rain season at two sites in Busia and Kakamega Counties. Data was collected on emergence, off types, lodging, ear aspect and abnormalities, incidence and severity of diseases and yield. Seeds from local market had significantly higher emergence of up to $66 \%$ while the farm saved seeds and local market resulted in the highest percentage of off-types of up to $18 \%$. Crop from farm saved seed and seeds from the local market showed high susceptibility to stalk lodging, ear abnormalities and high levels of diseases. Diseases detected include northern leaf blight, gray leaf spot, rust, brown spot, downy mildew, and ear rots. The study showed that though informal seeds had high plant establishment, they have high level of off types, are susceptible to lodging, diseases and low yields. Therefore, farmer should be encouraged to use certified or improved seeds to enhance crop productivity.
\end{abstract}

Keywords: disease, emergence, incidence, severity, maize, seed sources, yield

\section{Introduction}

Maize is the main food crop in Africa (Westengen et al., 2014) but yields remain low below $2,000 \mathrm{~kg} \mathrm{ha}^{-1}$ compared to average yields of 7,000 to $10,000 \mathrm{~kg} \mathrm{ha}^{-1}$ in other regions like the United States of America (Huang et al., 2011). Maize productivity is low due to several reasons including poor adoption of improved agricultural technologies and use of low quality farm-saved seeds by small scale farmers, who constitute the majority of food producers. The farm saved seeds make up to $80-90 \%$ of the available planting materials (Bruijin et al., 1994, Wright et al., 1994; Delouche, 1982). Farmer saved seeds are of low physiological quality and are usually contaminated with noxious weed seeds and seed borne diseases leading to low field emergence, reduced crop vigor and low yield (Sperling, 2001; Mahender et al., 2015). Louwaars et al. (1997) reported that good quality seed is a crucial input for plant productivity and unique way to maximize on other agricultural inputs.

Seed quality refers to it trueness to varietal type, physical attributes, ability to germinate, vigor and freedom from seed borne diseases. Low quality seeds result in defective seedlings, reduced crop stand, low crop establishment and infection with pests and diseases in the field (Coutts et al., 2008; IRRI, 2013). Therefore, frequent use of low quality seeds by small holder farmers leads to decline in maize productivity (Zegeye et al., 2014). In contrast, the use of certified seeds from the formal system results in crops with low incidence of pests, diseases and abiotic stresses and high yield (Zegeye et al., 2014; Alemu, 2015).

Foliage diseases affecting maize production include downy mildew (Sclerophthora spp.), common rust (Puccinia sorghi), turcicum leaf blight (Exserohilum turcicum), grey leaf spot (Cercospora zeae-maydis), stalk rot and ear rots (Fusarium spp and Diplodia spp). Common virus diseases affecting maize include maize streak virus (MSV) and maize lethal necrosis (MLN) (Tripathi et al., 2011; Wangai et al., 2012). Most of these diseases are 
transmitted through seed and yield losses due to these diseases are estimated to be up to $20 \%$ depending with the disease and the environmental conditions (Mueller et al., 2016).

Accessibility to certified quality seed by small-scale producers is a challenge in most African countries (COMESA, 2014) and therefore, adoption of improved seeds remains low at less than 10\% (Langyintuo, 2009). The certified seeds produced under the formal sector go through standards and regulations which differ from country to country. The distinctiveness, uniformity, and stability (DUS) and the value for cultivation and use (VCU) tests are conducted for a variety to be admitted for commercialization (ECAPAPA, 2003). The test, which is also referred to as the National Performance Trial (NPT), should be conducted in at least five to six locations. This makes it expensive for a seed company to release a variety (Setimela \& Mwangi, 2009). Use of high quality and clean seeds is likely to increase yields by up to 50\% (Atilaw, 2010; Asea et al., 2010; CTA, 2014; Gebremedhim, 2015; Mew \& Gonzales, 2002). The study was conducted to determine the effect of farm saved maize seeds on incidence and severity of foliage diseases.

\section{Materials and Methods}

\subsection{Experimental Design and Layout}

The field experiments were conducted during 2017 long rain season in Busia and at the Kenya Agricultural and Livestock Research Organization (KALRO) in Kakamega County. Seeds of maize varieties evaluated included farmer saved landraces Sipindi and Panadol and from local market, farmer saved Duma 43 and IR/Kayongo from and from Agrovet shops. Each variety was planted in $3 \mathrm{~m} \times 4 \mathrm{~m}$ plots separated by $1 \mathrm{~m}$ and laid out in a randomized complete block design with three replicates. Two seeds per hill were planted at a spacing of $75 \mathrm{~cm} \times$ $30 \mathrm{~cm}$ between and within rows, respectively. Two guard rows were installed around the experiment in both sites. Fertilization was done with $10 \mathrm{~g}$ diammonium phosphate (DAP) per hill at planting followed by top dressing with calcium ammonium phosphate (CAN) at the rate of $10 \mathrm{~g}$ per hill when the plants were about $45 \mathrm{~cm}$ tall (Muiru et al., 2015; Qasim et al., 2010; Halidu et al., 2014). Data was collected on emergence, viral diseases, off types, incidence and severity of fungal diseases, number of cobs per plant, cobs aspect and weight, and yield.

\subsection{Assessment of Agronomic Parameters}

The agronomic parameters recorded included emergence, dead seed, rotten seeds, lodging and ear aspects. Emergence was determined after 50\% seedlings had two fully expanded leaves (Kieran, 2009; Ogunniyan \& Olakojo, 2015). Dead seeds were assessed by checking hills where the seed did not emerge whether it was absent or rotten. Off-types crops were assessed in each plot by observing the morphological characteristics of the crop including Leaves shapes, tassel coloration at the base glume, silks color, plant length, ear and kernel coloration (Hipi et al., 2013; UPOV, 2009; Yadav \& Singh, 2010). Root and stalk lodging were recorded every month and at harvest as a percentage of the lodged plant over the total number of plants in each plot. Ear aspect was scored by on a scale of 1-5, where 1 is clean, uniform, large, and well-filled and 5 is rotten, variable, small, and partially filled ears (Adebayo \& Menkir, 2015).

\subsection{Assessment of Incidence and Severity of Foliage Diseases}

Disease incidence was based on visual symptoms while assessment of the major maize viral diseases including maize streak virus and maize lethal necrosis, was carried out three times at vegetative stage during the season. Inspection was done as described by CIMMYT (2004) at vegetative stage growth at V5, V10, VT before pollen development and R2, and at harvest. Incidence was determined as the percentage of the plants infected over the total number of plants in each plot. Incidence of viral diseases was determined on all plants in the 3 intermediate rows in each plot as described by Bosque-Perez et al. (1998). Severity was determined on 6 randomly selected plants showing disease symptoms. Two plant were selected in each of the three inner rows in each plot and the disease scored on a scale of 0-5 (Nwanosike et al., 2015; Reid et al., 1996), where, $0=$ no symptoms, $1=$ very slight infection, one or two restricted lesion on lower leaves; 2 = slight infections, 9-15 lesions on lower leaves; 3 moderate number of lesions on lower leaves; $4=$ abundant lesions on lower and middle leaves which extend to upper leaves; and $5=$ abundant infections, no formation of cobs, plant dying prematurely from the disease. Severity of ear rot was scored on rating scale of 1-7 (Shekhar \& Kumar, 2012), where $1=$ no infection, $2=1$ to $3 \%, 3=4$ to $10 \%, 4=11$ to $25 \%, 5=26$ to $50 \%, 6=51$ to $75 \%$ and $7=$ over $75 \%$ of ear infected.

\subsection{Assessment of Yield and Yield Components}

The crop was harvested at physiological maturity by removing the husks from the ears. The number of ears from each plot were counted and the following data collected: number of ears per plant, number of ears with abnormalities and total cob weight per plot. Ear abnormality was determined as incomplete kernel tip set, poor tip fill, bouquet ears, exposed ear tip, extended ear leaves, tassel ears and zipper ears. The data on the number of 
ears in each category was converted to percentage by dividing by the total number of ear in each plot, multiplied by 100 . Grain yield was determined in $\mathrm{kgha}^{-1}$ based on weight of unshelled cobs at physiological maturity, considering the commercial grain moisture of $12.5 \%$ and shelling factor of $80 \%$ as follows (Adebayo \& Menkir, 2015):

$$
\mathrm{GY}\left(\frac{\mathrm{Kg}}{\mathrm{ha}}\right)=\mathrm{WW} \times \frac{100-\mathrm{FM}}{100-12.5} \times \frac{80}{100} \times \frac{10,000}{\mathrm{US}}
$$

Where, GY is Grain yield, WW the wet weight, FM the field moisture and US the Useful surface.

\subsection{Data Analysis}

Data collected were subjected to analysis of variance (ANOVA) using GENSTAT $15^{\text {th }}$ edition. Mean separation was done using Fischer's protected Least Significant Difference (LSD) at 5\% level of significance.

\section{Results}

\subsection{Emergence and Plant Lodging}

There was significant variation $(\mathrm{p} \leq 0.05)$ in $50 \%$ emergence of the different seed sources between the sites. The result showed higher emergence percentage at Busia compared to Kakamega. Emergence percentage varied significantly among the seed sources at $\mathrm{p} \leq 0.05$ (Table 1). Uncertified seeds from farmer-saved and local market had about $43 \%$ emergence over the certified seed from agro vet shops. Panadol from local market had the highest emergence rate of about $63 \%$ while the certified IR/Kayongo from agro vet had the lowest in both sites. Dead seeds showed significant statistical variation in Busia. Certified seeds from agro vet shops showed high incidence of dead seeds of about $26 \%$ over uncertified seeds from farmer-saved and local market seeds. However, IR/Kayongo from agro-vet shop had the highest percentage of dead seeds of about $33 \%$ across the sites. Rotten seeds varied between the sites. There was no significant difference at Busia while at Kakamega, the result showed significant variations in percentage of rotten seeds among the seed sources. Certified seeds from agro vet shops had the highest percentage of rotten seeds of about $20 \%$ compared to uncertified seeds from farmer-saved and local market seeds. However, IR/Kayongo from agro-vet shop had the highest percentage of rotten seeds in both sites (Table 1).

Table 1. Percentage emergence, dead and rotten seeds of different seed sources in Busia and Kakamega sites

\begin{tabular}{llllllll}
\hline \multirow{2}{*}{ Seed sources } & \multicolumn{3}{c}{ Busia } & & \multicolumn{3}{c}{ Kakamega } \\
\cline { 2 - 3 } \cline { 6 - 7 } & Emergence & Dead seeds & Seed rot & & Emergence & Dead seeds & Seed rot \\
\hline Panadol local Market & $65.8_{\mathrm{a}}$ & $16.4_{\mathrm{bc}}$ & 12.1 & & $60.3_{\mathrm{a}}$ & 16.4 & $11.5_{\mathrm{b}}$ \\
Sipindi farmer saved & $56.4_{\mathrm{b}}$ & $16.7_{\mathrm{bc}}$ & 14.2 & & $53.3_{\mathrm{ab}}$ & 23.3 & $12.4_{\mathrm{b}}$ \\
IR/Kayo farmer saved & $55.8_{\mathrm{b}}$ & $18.2_{\mathrm{bc}}$ & 16.1 & & $39.4_{\mathrm{bc}}$ & 23.3 & $18.2_{\mathrm{b}}$ \\
Sipindi local market & $52.4_{\mathrm{b}}$ & $18.8_{\mathrm{bc}}$ & 14.2 & & $53.3_{\mathrm{ab}}$ & 21.5 & $15.2_{\mathrm{b}}$ \\
Duma 43 farmer saved & $52.4_{\mathrm{b}}$ & $20.3_{\mathrm{b}}$ & 15.5 & & $48.8_{\mathrm{ab}}$ & 17.9 & $18.5_{\mathrm{b}}$ \\
Panadol farmer saved & $50.0_{\mathrm{b}}$ & $19.4_{\mathrm{bc}}$ & 17.6 & & $42.4 \mathrm{a}_{\mathrm{bc}}$ & 23.3 & $19.1_{\mathrm{ab}}$ \\
Duma 43 Agrovet & $49.7_{\mathrm{b}}$ & $15.2_{\mathrm{c}}$ & 12.4 & & $54.2_{\mathrm{ab}}$ & 10.3 & $14.2_{\mathrm{b}}$ \\
IR/Kayo Agrovet & $27.9_{\mathrm{c}}$ & $30.6_{\mathrm{a}}$ & 25.8 & & $24.2_{\mathrm{c}}$ & 35.5 & $26.4_{\mathrm{a}}$ \\
Grand Mean & 55.9 & 19.4 & 16.0 & & 47.0 & 21.9 & 16.9 \\
LSD (p $\leq 0.05$ ) & 21.5 & 5.1 & 9.8 & & 20.4 & 23.5 & 7.7 \\
CV\% & 21.9 & 15.0 & 35.1 & & 24.7 & 61.2 & 25.8 \\
\hline
\end{tabular}

The result showed significant variation at $\mathrm{p} \leq 0.05$ in off-types among the seeds from the various sources in both sites. Uncertified seeds from farmer saved and local market had high number of off-types of about $9 \%$ in contrast to certified seeds from agro vet shops having about 5\% at Busia site while at Kakamega, the informal seeds had about $17 \%$ of off-type and formal seeds from agro vet shops had about $3 \%$. However, Sipindi from farmer-saved had high number of off-types crops across the sites (Table 2). Stalk lodging did not show significant variation between the sites. At both sites, uncertified seeds from farmer saved and local market had high incidence of stalk lodging at $49.4 \%$ over certified seeds from agro vet shops (Table 2 ). 
Table 2. Percentage plant lodging of the seed from various sources at Busia and Kakamega sites

\begin{tabular}{|c|c|c|c|c|c|c|}
\hline \multirow{2}{*}{ Seed sources } & \multicolumn{3}{|c|}{ Busia } & \multicolumn{3}{|c|}{ Kakamega } \\
\hline & Off-types & Root lodging & Stalk lodging & Off-types & Root lodging & Stalk lodging \\
\hline Panadol farmer saved & $10.6 \mathrm{a}$ & 7.7 & $11.5 \mathrm{ab}$ & $14.6 \mathrm{a}$ & 5.3 & $12.2 \mathrm{a}$ \\
\hline Duma 43 farmer saved & $10.4 \mathrm{ab}$ & 7.5 & $10.4 \mathrm{ab}$ & $15.3 \mathrm{a}$ & 6.1 & $12.9 \mathrm{a}$ \\
\hline Sipindi local market & $9.2 \mathrm{abc}$ & 6.4 & $14.1 \mathrm{a}$ & $15.3 \mathrm{a}$ & 5.8 & $12.2 \mathrm{a}$ \\
\hline Sipindi farmer saved & $8.6 a b c$ & 7.1 & $11.2 \mathrm{ab}$ & $21.9 \mathrm{a}$ & 6.8 & $11.7 \mathrm{a}$ \\
\hline IR/Kayongo farmer saved & $7.1 \mathrm{bc}$ & 8.1 & $10.9 \mathrm{ab}$ & $14.9 \mathrm{a}$ & 6.0 & $11.0 \mathrm{a}$ \\
\hline IR/Kayongo Agro vet & $6.7 \mathrm{c}$ & 7.6 & $12.1 \mathrm{ab}$ & $3.4 \mathrm{~b}$ & 5.4 & $12.2 \mathrm{a}$ \\
\hline Panadol local Market & $6.0 \mathrm{~cd}$ & 5.7 & $8.3 \mathrm{bc}$ & $18.9 \mathrm{a}$ & 5.3 & $11.3 \mathrm{a}$ \\
\hline Duma 43 Agro vet & $3.1 \mathrm{~d}$ & 7.9 & $3.7 \mathrm{c}$ & $2.7 \mathrm{~b}$ & 5.8 & $2.8 \mathrm{~b}$ \\
\hline Grand mean & 7.7 & 7.3 & 10.3 & 13.4 & 5.8 & 10.8 \\
\hline $\operatorname{LSD}(p \leq 0.05)$ & 3.4 & 2.7 & 5.3 & 10.3 & 2.0 & 2.5 \\
\hline $\mathrm{CV} \%$ & 24.8 & 21.5 & 29.4 & 43.9 & 19.7 & 13.3 \\
\hline
\end{tabular}

\subsection{Incidence and Severity of Diseases}

Major diseases observed included Seedling blight, maize streak, northern leaf blight, grey leaf spot, rust, diplodia, downy mildew, brown spot, eyespot and ear rot. Seedling blight, Northern leaf blight, and downy mildew were the commonest folia diseases affecting the maize from various sources in both Busia and Kakamega sites. Except at Busia, where maize streak virus, rust, brown spot which had significant differences compared to Kakamega where high incidences were observed in northern leaf blight, diplodia, grey leaf spot and downy mildew. There were no much variations in the incidence of seedling blight between the sites at $p \leq 0.05$. At both sites, certified seeds from agro vet shops had the highest incidence of seedling blight and maize streak virus. However, IR/Kayongo from agro vet shops was highly contaminated with seedling blight and maize streak virus at both sites (Table 3).

Table 3. Incidence of seedling blight and Maize Streak Virus of the seed sources at Busia and Kakamega sites

\begin{tabular}{|c|c|c|c|c|}
\hline \multirow{2}{*}{ Seed sources } & \multicolumn{2}{|c|}{ Busia } & \multicolumn{2}{|c|}{ Kakamega } \\
\hline & Seedling blight & Maize Streak Virus & Seedling blight & Maize Streak Virus \\
\hline IR Agrovet & $39.2_{\mathrm{a}}$ & $13.4_{a}$ & $46.3_{\mathrm{a}}$ & 11.4 \\
\hline Sipindi local market & $27.9_{\mathrm{b}}$ & $8.5_{\mathrm{bc}}$ & $31.7_{\mathrm{b}}$ & 0.0 \\
\hline Panadol local Market & $21.4_{b c}$ & $7.8_{\mathrm{bcd}}$ & $21.9_{\mathrm{bc}}$ & 0.0 \\
\hline Panadol farmer saved & $20.6_{\mathrm{bc}}$ & $10.6_{\mathrm{ab}}$ & $29.1_{\mathrm{bc}}$ & 0.0 \\
\hline Duma 43 farmer saved & $20.3_{\mathrm{bc}}$ & $9.8_{\mathrm{ab}}$ & $24.2_{\mathrm{bc}}$ & 2.4 \\
\hline IR farmer saved & $18.7_{\mathrm{bc}}$ & $4.8_{\mathrm{cd}}$ & $29.5_{\mathrm{bc}}$ & 5.3 \\
\hline Sipindi farmer saved & $18.4_{\mathrm{c}}$ & $8.2_{\mathrm{bc}}$ & $20.7_{\mathrm{c}}$ & 5.3 \\
\hline Duma 43 Agrovet & $7.9_{\mathrm{d}}$ & $3.3_{\mathrm{d}}$ & $6.5_{\mathrm{d}}$ & 0.0 \\
\hline Grand Mean & 21.8 & 8.0 & 26.2 & 31.4 \\
\hline $\operatorname{LSD}(p=0.05)$ & 9.4 & 4.9 & 10.1 & 10.4 \\
\hline $\mathrm{CV} \%$ & 24.7 & 33.4 & 22.0 & 18.8 \\
\hline
\end{tabular}

The result showed at both sites, highly significant variations of fungal foliage diseases incidence between the seed sources. At Busia site, uncertified seeds from farmer saved and local market were found to be more infected having high incidence of diseases including northern leaf blight (17\%), diplodia $(6 \%)$ rust $(4 \%)$, brown spot (3\%) while certified seeds from agro vet shops had high incidence of downy mildew only. At Kakamega, the result revealed a reverse situation whereby the certified seeds from agro vet shops had a high level of diseases incidence of about $20 \%$ for northern leaf blight, grey leaf spot at $6 \%$ incidence, diplodia $12 \%$ and down mildew $6 \%$. Across the sites, the result showed that certified seeds from agro vet shops had high incidence of northern leaf blight (17\%), grey leaf spot (3\%), downy mildew (3\%) while incidence of rust (4\%), brown spot (4\%) and eye spot (3\%) were highly found in uncertified seeds from farmer saved and local market (Table 4). 
Severity score of fungal diseases was significantly different between the sites and among the seed sources. At Busia site, diplodia was significantly different among the seeds sources whereas, at Kakamega, variations were found in northern leaf blight, grey leaf spot, diplodia, and eyespot. Diplodia was found to be severe in uncertified seeds at Busia site while at Kakamega site, high severity scores of northern leaf blight, grey leaf spot were found in certified seeds from agro vet shops (Table 5).

Incidence and severity scores of ear rot were found to be highly significant among the seeds sources at Busia site, whereas at Kakamega there was non-significant difference. Farmer saved and local market seeds had high incidence of $10.5 \%$ and severity score of up to 6 indicating 51 to $75 \%$ infection on ears compared to certified seeds from agro vet shops. However, local market, although had high plant establishment as indicated in Table 4.1, had high incidence and severity of ear rot (Table 6).

Table 4. Incidence of fungal diseases of seed sources in Busia and Kakamega sites

\begin{tabular}{|c|c|c|c|c|c|c|c|}
\hline Seed sources & Northern leaf blight & Gray leaf spot & Diplodia & Rust & Brown spot & Downy mildew & Eyespot \\
\hline \multicolumn{8}{|l|}{ Busia } \\
\hline IR Agrovet & $25.2_{\mathrm{a}}$ & 0.0 & $7.9_{\mathrm{ab}}$ & $4.4_{\mathrm{b}}$ & $3.4_{\mathrm{bc}}$ & $0.0_{\mathrm{b}}$ & 0.0 \\
\hline Sipindi local market & $22.7_{\mathrm{a}}$ & 0.0 & $8.5_{\mathrm{a}}$ & $3.9 \mathrm{~b}$ & $7.8_{\mathrm{a}}$ & $0.6_{\mathrm{b}}$ & 0.0 \\
\hline Duma 43 farmer saved & $22.3_{\mathrm{ab}}$ & 0.0 & $4.9_{\mathrm{bcd}}$ & $3.7 \mathrm{~b}$ & $2.4_{\text {bcd }}$ & $0.6_{\mathrm{b}}$ & 0.0 \\
\hline IR farmer saved & $19.1_{\mathrm{b}}$ & 0.0 & $5.4_{\text {abcd }}$ & $6.6_{\mathrm{a}}$ & $3.0_{\mathrm{bcd}}$ & $0.0_{\mathrm{b}}$ & 0.0 \\
\hline Sipindi farmer saved & $14.6_{\mathrm{c}}$ & 0.0 & $4.1_{\mathrm{cd}}$ & $1.8_{\mathrm{c}}$ & $1.8_{\mathrm{cd}}$ & $1.1_{\mathrm{ab}}$ & 0.0 \\
\hline Panadol local Market & $13.1_{\mathrm{c}}$ & 0.0 & $5.7 \mathrm{abc}$ & $1.4_{\mathrm{c}}$ & $1.7_{\mathrm{cd}}$ & $0.0_{\mathrm{b}}$ & 0.0 \\
\hline Panadol farmer saved & $12.3_{\mathrm{c}}$ & 0.0 & $5.9_{\mathrm{abc}}$ & $3.9_{\mathrm{b}}$ & $3.9_{\mathrm{b}}$ & $0.0_{\mathrm{b}}$ & 0.0 \\
\hline Duma 43 Agro vet & $3.9_{\mathrm{d}}$ & 0.0 & $1.9 \mathrm{~d}$ & $0.0_{\mathrm{c}}$ & $1.3_{\mathrm{d}}$ & $1.9_{\mathrm{a}}$ & 0.0 \\
\hline Grand mean & 7.1 & 0.0 & 5.5 & 3.2 & 3.2 & 0.5 & 0.0 \\
\hline $\operatorname{LSD}(p \leq 0.05)$ & 2.3 & 0.0 & 3.5 & 1.9 & 1.98 & 1.16 & 0.0 \\
\hline $\mathrm{CV} \%$ & 18.7 & 0.0 & 36.5 & 34.4 & 35.6 & 122.9 & 0.0 \\
\hline \multicolumn{8}{|l|}{ Kakamega } \\
\hline IR Agro vet & $29.8 \mathrm{a}$ & $12.2_{\mathrm{a}}$ & $21.8_{\mathrm{a}}$ & 6.7 & 3.8 & $10.4_{a}$ & 4.2 \\
\hline Sipindi local market & $12.6_{\mathrm{b}}$ & $7.1_{\mathrm{b}}$ & $10.5 \mathrm{~b}$ & 10.6 & 3.7 & $0.0_{\mathrm{b}}$ & 10.7 \\
\hline Duma 43 farmer saved & $12.2_{\mathrm{b}}$ & $2.3_{\mathrm{c}}$ & $6.9_{\mathrm{bc}}$ & 5.5 & 2.3 & $0.8_{\mathrm{b}}$ & 6.1 \\
\hline IR farmer saved & $9.9_{\mathrm{bc}}$ & $4.9_{\mathrm{b}}$ & $11.0_{\mathrm{b}}$ & 3.2 & 1.1 & $1.0_{\mathrm{b}}$ & 5.9 \\
\hline Sipindi farmer saved & $4.4_{c}$ & $0.0_{\mathrm{c}}$ & $7.8_{\mathrm{bc}}$ & 4.7 & 7.7 & $0.6_{\mathrm{b}}$ & 5.6 \\
\hline Panadol local Market & $7.7 \mathrm{bc}$ & $2.4_{\mathrm{c}}$ & $11.4_{b}$ & 6.9 & 4.2 & $2.5 \mathrm{~b}$ & 5.2 \\
\hline Panadol farmer saved & $6.8_{\mathrm{bc}}$ & $1.6_{\mathrm{c}}$ & $13.5_{\mathrm{b}}$ & 0.8 & 6.4 & $4.6_{\mathrm{ab}}$ & 1.4 \\
\hline Duma 43 Agro vet & $9.5_{\mathrm{bc}}$ & $0.0_{\mathrm{c}}$ & $2.0_{\mathrm{c}}$ & 1.2 & 1.1 & $1.1_{\mathrm{b}}$ & 1.1 \\
\hline Grand mean & 11.6 & 3.8 & 10.6 & 4.9 & 3.8 & 2.6 & 5 \\
\hline $\operatorname{LSD}(p \leq 0.05)$ & 6.92 & 2.39 & 8.17 & 8.93 & 5.62 & 6.09 & 5.64 \\
\hline $\mathrm{CV} \%$ & 34 & 35.8 & 44 & 103 & 84.7 & 132.3 & 64.1 \\
\hline
\end{tabular}


Table 5. Severity scores of fungal diseases of seed sources in Busia and Kakamega sites

\begin{tabular}{|c|c|c|c|c|c|c|c|}
\hline Seed sources & Northern leaf blight & Gray leaf spot & Diplodia & Rust & Brown spot & Downy mildew & Eyespot \\
\hline \multicolumn{8}{|l|}{ Busia } \\
\hline IR Agrovet & 1.3 & 0.0 & $1.7 \mathrm{bc}$ & 1.0 & 0.3 & 1.3 & 0.0 \\
\hline Sipindi local market & 1.7 & 0.0 & $2.3_{\mathrm{ab}}$ & 1.0 & 2.0 & 1.3 & 0.0 \\
\hline Duma 43 farmer saved & 1.7 & 0.0 & $2.3_{\mathrm{ab}}$ & 1.3 & 1.0 & 1.3 & 0.0 \\
\hline IR farmer saved & 1.3 & 0.0 & $1.7 \mathrm{bc}$ & 2.3 & 1.7 & 1.0 & 0.0 \\
\hline Sipindi farmer saved & 1.7 & 0.0 & $2.3_{\mathrm{ab}}$ & 1.7 & 2.0 & 1.3 & 0.0 \\
\hline Panadol local Market & 1.3 & 0.0 & $3.3_{\mathrm{a}}$ & 1.7 & 1.0 & 1.0 & 0.0 \\
\hline Panadol farmer saved & 0.7 & 0.0 & $2.7_{\mathrm{ab}}$ & 1.7 & 2.0 & 1.0 & 0.0 \\
\hline Duma 43 Agro vet & 1.3 & 0.0 & $0.7_{\mathrm{c}}$ & 0.3 & 0.7 & 1.7 & 0.0 \\
\hline Grand mean & 1.4 & 0.0 & 2.12 & 1.4 & 1.3 & 1.3 & 0.0 \\
\hline $\operatorname{LSD}(\mathrm{p} \leq 0.05)$ & 1.5 & 0.0 & 1.2 & 1.5 & 1.7 & 1.1 & 0.0 \\
\hline $\mathrm{CV} \%$ & 63.2 & 0.0 & 32.3 & 63.7 & 70.6 & 48.2 & 0.0 \\
\hline \multicolumn{8}{|l|}{ Kakamega } \\
\hline IR Agrovet & $3.7 \mathrm{a}$ & $3.7_{\mathrm{a}}$ & $2.7_{\mathrm{ab}}$ & 0.7 & 0.3 & 2.0 & $0.7_{\mathrm{b}}$ \\
\hline Sipindi local market & $3.7_{\mathrm{a}}$ & $2.7_{\mathrm{b}}$ & $2.3_{\mathrm{ab}}$ & 2.3 & 1.3 & 1.0 & $3.0_{\mathrm{a}}$ \\
\hline Duma 43 farmer saved & $2.7_{\mathrm{b}}$ & $1.3_{\mathrm{c}}$ & $3.3_{\mathrm{a}}$ & 1.3 & 2.0 & 1.3 & $2.0_{\mathrm{ab}}$ \\
\hline IR farmer saved & $2.7_{\mathrm{b}}$ & $2.3_{\mathrm{b}}$ & $2.3_{\mathrm{ab}}$ & 1.0 & 0.7 & 1.3 & $1.3_{\mathrm{b}}$ \\
\hline Sipindi farmer saved & $1.3_{\mathrm{d}}$ & $0.0_{\mathrm{d}}$ & $2.0_{\mathrm{bc}}$ & 1.7 & 2.0 & 1.3 & $2.0_{\mathrm{ab}}$ \\
\hline Panadol local Market & $1.7_{\mathrm{cd}}$ & $1.3_{\mathrm{c}}$ & $3.0_{\mathrm{ab}}$ & 2.3 & 1.3 & 2.3 & $2.0_{\mathrm{ab}}$ \\
\hline Panadol farmer saved & $1.3_{\mathrm{d}}$ & $0.3_{\mathrm{d}}$ & $2.0_{\mathrm{bc}}$ & 1.7 & 1.0 & 1.7 & $0.7_{\mathrm{b}}$ \\
\hline Duma 43 Agro vet & $2.3_{\mathrm{bc}}$ & $0.0_{\mathrm{d}}$ & $1.0_{\mathrm{c}}$ & 0.3 & 1.3 & 1.7 & $0.7_{\mathrm{b}}$ \\
\hline Grand mean & 2.4 & 1.5 & 2.3 & 1.2 & 1.3 & 1.6 & 1.5 \\
\hline $\operatorname{LSD}(\mathrm{p} \leq 0.05)$ & 0.8 & 0.9 & 1.2 & 2.0 & 1.3 & 1.2 & 1.4 \\
\hline CV\% & 19.2 & 35.1 & 28.3 & 98.8 & 57.0 & 43.6 & 52.2 \\
\hline
\end{tabular}

Table 6. Incidence and severity score of ear rot of the different seed sources in Busia and Kakamega sites

\begin{tabular}{llllll}
\hline \multirow{2}{*}{ Seed sources } & \multicolumn{2}{c}{ Busia } & & \multicolumn{2}{c}{ Kakamega } \\
\cline { 2 - 3 } \cline { 5 - 6 } \cline { 5 - 5 } Panadol local Market & Incidence & Severity scores & & Incidence & Severity scores \\
Sipindi local market & $14.2_{\mathrm{a}}$ & $5.3_{\mathrm{ab}}$ & & 44.4 & 7.0 \\
Duma 43 farmer saved & $11.0_{\mathrm{b}}$ & $6.3_{\mathrm{a}}$ & & 49.6 & 7.0 \\
Panadol farmer saved & $10.2_{\mathrm{bc}}$ & $4.3_{\mathrm{b}}$ & & 55.5 & 7.0 \\
IR Agro vet & $9.4_{\mathrm{bc}}$ & $5.3_{\mathrm{ab}}$ & & 54.3 & 6.0 \\
IR farmer saved & $9.3_{\mathrm{bc}}$ & $3.7_{\mathrm{b}}$ & & 53.9 & 5.0 \\
Sipindi farmer saved & $8.3 \mathrm{~b}_{\mathrm{cd}}$ & $4.0_{\mathrm{b}}$ & & 51.7 & 6.0 \\
Duma 43 Agro vet & $7.9_{\mathrm{cd}}$ & $4.0_{\mathrm{b}}$ & & 34.7 & 6.0 \\
Grand mean & $5.7_{\mathrm{d}}$ & $1.7_{\mathrm{c}}$ & & 52.8 & 7.0 \\
LSD ( $\mathrm{p}=0.05)$ & 9.5 & 4.0 & & 49.6 & 6.0 \\
CV\% & 3.0 & 2.0 & 25.9 & 16.1 & 1.6 \\
\hline
\end{tabular}

\subsection{Yield and Yield Components}

At both sites, ears had developed some abnormalities including incomplete kernel tip set, poor tip fill, bouquet ears, exposed ear tip, tassel ear and zipper ear. There were significant variations between the seed sources in ear abnormalities at the two sites. Uncertified seeds from farmer saved and local market had high number of ears having abnormalities at both sites including incomplete kernel tip set, poor tip fill, bouquet ears, tassel ear and zipper ear, while the certified seeds from agro vet shops had high number of exposed ear tip and ears with extended leaves at both sites (Table 7). There was no significant variation in number of ears per plant in both sites while the result showed significant variation in ear length and grain yield in the two sites. Seeds from agro vet shops were found to have high ear length and grain yield at both sites depending on the variety type. 
However, Duma 43 from agro-vet shop performed with high ear length and grain yield of up to $6.461 \mathrm{~kg} \mathrm{ha}^{-1}$ (Table 8).

Table 7. Percentage Ear abnormalities of various seed sources in Busia and Kakamega sites

\begin{tabular}{|c|c|c|c|c|c|c|c|}
\hline Seed sources & Incomplete kernel tip set & Poor tip fill & Bouquet ear & Exposed ear tip & Ear with extended leaves & Tassel ear & Zipper ear \\
\hline \multicolumn{8}{|l|}{ Busia } \\
\hline Sipindi local market & $17.7 \mathrm{a}$ & 21.3 & $0.0_{\mathrm{d}}$ & $8.5_{\text {bcd }}$ & $5.8 \mathrm{bc}$ & $0.0_{\mathrm{b}}$ & $7.8_{\mathrm{ab}}$ \\
\hline Panadol local Market & $16.3_{\mathrm{ab}}$ & 24 & $6.6_{\mathrm{a}}$ & $12.4_{\mathrm{a}}$ & $2.9_{\mathrm{cd}}$ & $0.0_{\mathrm{b}}$ & $3.4_{d}$ \\
\hline Sipindi farmer saved & $12.3_{\mathrm{bc}}$ & 18 & $1.2_{\mathrm{c}}$ & $6.7_{\mathrm{d}}$ & $0.6_{d}$ & $1.2_{\mathrm{b}}$ & $3.7_{d}$ \\
\hline Panadol farmer saved & $11.0_{\mathrm{cd}}$ & 20.7 & $3.3_{\mathrm{b}}$ & $10.1_{\mathrm{abc}}$ & $5.6_{\mathrm{bc}}$ & $0.0_{\mathrm{b}}$ & $8.3_{\mathrm{a}}$ \\
\hline Duma 43 farmer saved & $10.3_{\mathrm{cd}}$ & 18.3 & $0.0_{\mathrm{d}}$ & $11.4_{\mathrm{ab}}$ & $4.6_{\mathrm{bc}}$ & $0.0_{\mathrm{b}}$ & $4.6_{\mathrm{cd}}$ \\
\hline IR farmer saved & $7.0_{\mathrm{de}}$ & 18 & $0.0_{\mathrm{d}}$ & $10.9_{\mathrm{ab}}$ & $7.7_{\mathrm{ab}}$ & $3.7 \mathrm{a}$ & $3.6_{\mathrm{d}}$ \\
\hline Duma 43 Agrovet & $4.0_{\mathrm{e}}$ & 18.7 & $0.0_{\mathrm{d}}$ & $7.1_{\mathrm{cd}}$ & $6.5_{\mathrm{ab}}$ & $0.0_{\mathrm{b}}$ & $4.3_{\mathrm{d}}$ \\
\hline IR Agrovet & $4.0_{\mathrm{e}}$ & 17.7 & $0.0_{\mathrm{d}}$ & $9.3_{\text {bed }}$ & $9.3_{\mathrm{a}}$ & $0.0_{\mathrm{b}}$ & $6.2_{\mathrm{bc}}$ \\
\hline Grand mean & 10.3 & 19.6 & 1.4 & 9.6 & 5.4 & 0.6 & 5.2 \\
\hline $\operatorname{LSD}(p \leq 0.05)$ & 4.4 & 5.4 & 0.99 & 3.1 & 3.3 & 1.4 & 1.7 \\
\hline CV\% & 24.1 & 15.8 & 41.1 & 18.3 & 35.3 & 128.4 & 18.6 \\
\hline \multicolumn{8}{|l|}{ Kakamega } \\
\hline Sipindi local market & $37.9 \mathrm{~b}$ & $46.2 \mathrm{~b}$ & 8.9 & $15.9_{\mathrm{bc}}$ & $10.6_{\mathrm{b}}$ & $0.0_{\mathrm{c}}$ & $10.7_{\mathrm{a}}$ \\
\hline Panadol local Market & $41.5 \mathrm{a}$ & $42.5_{\mathrm{bc}}$ & 8.4 & $10.9_{\mathrm{cd}}$ & $7.3_{b}$ & $0.6_{\mathrm{bc}}$ & $4.9 \mathrm{~b}$ \\
\hline Sipindi farmer saved & $25.7_{d}$ & $35.4_{c}$ & 6 & $3.7_{\mathrm{e}}$ & $6.4_{b}$ & $1.3_{\mathrm{bc}}$ & $3.7_{\mathrm{b}}$ \\
\hline Panadol farmer saved & $29.6_{\mathrm{c}}$ & $54.4_{a}$ & 8.5 & $10.5_{\mathrm{cd}}$ & $15.3_{\mathrm{ab}}$ & $0.0_{\mathrm{c}}$ & $10.7_{\mathrm{a}}$ \\
\hline Duma 43 farmer saved & $16.3_{\mathrm{e}}$ & $46.9_{\mathrm{ab}}$ & 8.6 & $8.8_{\mathrm{de}}$ & $6.9 \mathrm{~b}$ & $3.4_{a}$ & $6.0_{\mathrm{ab}}$ \\
\hline IR farmer saved & $36.1_{\mathrm{b}}$ & $48.2_{\mathrm{ab}}$ & 5.3 & $21.1_{\mathrm{b}}$ & $11.3_{\mathrm{b}}$ & $1.8_{\mathrm{ab}}$ & $2.6_{\mathrm{b}}$ \\
\hline Duma 43 Agrovet & $11.8_{\mathrm{f}}$ & $16.6_{\mathrm{d}}$ & 3.7 & $27.6_{a}$ & $5.8_{\mathrm{b}}$ & $1.3_{\mathrm{bc}}$ & $1.5_{\mathrm{b}}$ \\
\hline IR Agrovet & $11.1_{\mathrm{f}}$ & $17.4_{d}$ & 6.3 & $9.6_{d}$ & $24.9 \mathrm{a}$ & $0.0_{\mathrm{c}}$ & $5.2_{\mathrm{ab}}$ \\
\hline Grand mean & 26.3 & 38.5 & 7 & 13.5 & 11.1 & 1 & 5.7 \\
\hline $\operatorname{LSD}(p \leq 0.05)$ & 3.3 & 7.42 & 4.75 & 5.9 & 10.7 & 1.7 & 5.7 \\
\hline $\mathrm{CV} \%$ & 7.1 & 11 & 39 & 25 & 55.3 & 94 & 57.1 \\
\hline
\end{tabular}

Table 8. Ear length and grain yield $\left(\mathrm{kg} \mathrm{ha}^{-1}\right)$ of various seed sources in Busia and Kakamega sites

\begin{tabular}{llllll}
\hline \multirow{2}{*}{ Seed sources } & \multicolumn{3}{c}{ Busia } & & \multicolumn{2}{c}{ Kakamega } \\
\cline { 2 - 3 } \cline { 5 - 6 } & Ear length & Grain Yield kg ha ${ }^{-1}$ & & Ear length & Grain Yield kg ha $^{-1}$ \\
\hline Duma 43 Agrovet & $21.3_{\mathrm{a}}$ & $6543_{\mathrm{a}}$ & & $19.5_{\mathrm{a}}$ & $6379_{\mathrm{a}}$ \\
Panadol farmer saved & $18.3_{\mathrm{b}}$ & $5733_{\mathrm{ab}}$ & & $16.4_{\mathrm{c}}$ & $3934_{\mathrm{b}}$ \\
Duma 43 farmer saved & $17.8_{\mathrm{bc}}$ & $5564_{\mathrm{ab}}$ & & $18.9_{\mathrm{ab}}$ & $4321_{\mathrm{b}}$ \\
Sipindi local market & $17.6_{\mathrm{bc}}$ & $4956_{\mathrm{b}}$ & & $19.4_{\mathrm{a}}$ & $3916_{\mathrm{b}}$ \\
IR farmer saved & $17.4_{\mathrm{bc}}$ & $5407_{\mathrm{ab}}$ & & $17.8_{\mathrm{abc}}$ & $3734_{\mathrm{b}}$ \\
Panadol local Market & $17.1_{\mathrm{bc}}$ & $6115_{\mathrm{ab}}$ & & $17.6_{\mathrm{bc}}$ & $4270_{\mathrm{b}}$ \\
Sipindi farmer saved & $17.1_{\mathrm{bc}}$ & $5166_{\mathrm{b}}$ & & $18.0_{\mathrm{abc}}$ & $4157_{\mathrm{b}}$ \\
IR Agrovet & $16.5_{\mathrm{c}}$ & $3061_{\mathrm{c}}$ & & $17.4_{\mathrm{bc}}$ & $3094_{\mathrm{b}}$ \\
Grand mean & 17.9 & 5318 & & 18.1 & 4225.6 \\
LSD $(\mathrm{p} \leq 0.05)$ & 1.64 & 1203.9 & & 1.8 & 1578.1 \\
$\mathrm{CV} \%$ & 5.2 & 12.9 & & 5.5 & 21.3 \\
\hline
\end{tabular}

\section{Discussion}

The uncertified seed from local market and farmer saved had high plant establishment while both market and farmer saved seeds highly lodged and counted high number of off-type crops. Dead seeds and rotten seed incidences were higher in certified seeds from agro vet shops. Similar study done by Bishaw et al. (2012) reported a higher level of germination rate in informal seeds compared to certified seeds. FAO (2016b) also 
reported an isolated case of certified seeds which failed to germinate. This is contrary to the finding of Sperling (2001) and Mahender (2015) who found that informal seeds are usually of low germination potential in field due to low quality. Also, IRRI (2013) and Coomes et al. (2015) reported that poor seed quality results in uneven seedling stands and more unhealthy seedlings. This is similar to other research by Paplomatas (2006), Niaz and Dawar (2009) which reported that seed quality refers to its ability to germinate.

Mostly, farmer saved seeds are selected from the previous harvest and maybe having the probability to emerge faster than the seeds stored for a time. Agro dealer may store their maize seeds for a long time as farmers rely on their own production. Wekesa et al. (2003) observed that improved cultivars do not have good storability compared to landraces, thus certified seeds lose their potential to germinate faster. Adetumbi and Olakoje (2010) also indicated that seed storage is critical for seed quality. It can have an impact on the whole feature of seeds quality attributes and contributes to seed ageing that decreases seed viability. Also, Bennett and Klich (2003) observed that good storage is necessary for seed fitness maintenance. Store environments must remain properly cleaned to prevent leftover contagions from diseased vegetable or seed material saved from previous harvest (Anteneh, 2015).

Seed transmitted diseases constitute the main challenge in the world concerning seed dissemination. They are the most causes of poor performance of the crop from field emergence up to the yield (Du et al., 2001; Rajput et al., 2005; Munkvol \& White, 2016; Mueller et al., 2016). In relation to seed testing, storage is important when testing date and planting date are far apart (Rao et al., 2006). Study done by Du et al. (2001), Rajput et al. (2005), Niaz and Dawar (2009), Paplomatas (2006) indicated that seed quality refers to it trueness to it varietal type, physical attribute. Same result was obtained by Sperling (2001) who reported that seeds from the informal system are inappropriate varieties. Badu et al. (2014) also reported that informal seeds do not go through guarantee standards and controlled production networks and therefore could be of poor quality.

Lodging sensitivity of the crops was possibly the effect of seed-borne pathogens observed in the seed samples after laboratory test which reduced resistance to stress due to low vigor. Presence of fungal seed borne pathogens decreases the germination potential and vigor of seeds thus reduces plant development especially when the environment is favorable for disease development (Botelho et al., 2013; Pathak \& Zaidi, 2013). Similar study by Badu (2007) also found that recycled seeds are most of the time infected with seed borne pathogens that can reduce crop resistance due to low vigor, can cause lodging, thus lead to low productivity. Poor seed quality results in defective seedling stands and more unhealthy plants in field (IRRI, 2013; FAO, 2009).

Uncertified seeds from farmer saved and local market showed high incidence and severity of fungal diseases though certified was highly susceptible to seedling blight and maize streak virus depending on the variety type. Similar study done by Tonu et al. (2017) found that fungal diseases intensity was highly expressive in farmer saved wheat than cleaned seeds (Bishaw et al., 2012). There was variation in number of fungal infections in farmer saved and local market seeds and this was highly expressed in Kakamega site. This situation may be explained by environment agro climatic difference whereby Kakamega site is relatively humid than Busia. This was also confirmed by Badu et al. (2007) who reported that seed borne fungi whereof, are usually severely manifested in an environment with high relative humidity. Fungal diseases develop better in zones with high humidity (Mueller et al., 2016; Munkvold \& White, 2016; Wise et al., 2016).

Fungal diseases were more expressed in farmer saved and local market seeds. Common diseases were northern leaf blight, grey leaf blight, rust, brown spot, diplodia, eyespot and ear rots. Though they were observed in all the material, they were severe in the uncertified seeds compared to the seed from agro vet shops. Du et al. (2001), Rajput et al. (2005), Niaz and Dawar, (2009), and Paplomatas (2006) reported that seed-transmitted diseases constitute the main challenge in the world concerning seed dissemination. They are the most causes of poor performance of the crop from field emergence up to the yield. Poor seed quality results in defective seedling stands and more unhealthy plants in the field reducing sensibly production and quality of end product (Dornbos, 1995; Powell et al., 1984; AGRA, 2017; Ali, 2016).

Farmer saved and local market seeds have developed ear abnormalities including incomplete kernel tip set, poor tip fill, bouquet ears, exposed ear tip, extended ear leaves, tassel ear and zipper ear compared to certified maize seed from the agro vet shops. Peter and Allen (2015) determined that abnormal ears are caused by multiple factors including temperature pressure (cold threat) during initial ear development which can cause bouquet ear; tillers (Suckers) when the growing point is destroyed or injured by hail, frost, flooding, herbicides, and automated damage can cause tassel ears. Poor ear tip fill and incomplete kernel tip set can occur when there is poor fertilization of ear tip ovules at silking. It can occur due to inadequate pollen supply caused by uneven crop 
development, herbicides, insect feeding and foliar diseases (Nielsen, 2006, 2009; Elmore et al., 2006; Thomison, 2007).

Uncertified seeds from farmer saved and local market seeds performed poorly compared to the certified seeds from agro vet shops which performance was dependent on the variety type. The certified Duma 43 from the agro vet shops performed well having low incidence and severity of diseases leading to high yield in both sites. Similar study done by Furtas (2016), and Joshi et al. (2016) indicated that certified seeds of newly developed cultivar are of high yield, diseases confrontation and stresses acceptance (Desalegn, 2017). Also, Sperling (2001) reported that farmer seeds are unsuitable varieties, contaminated, the seed quality attributes are below the standards limiting producers' crop output. Good quality of planting material is crucial for better production. It allows a rational estimation of farm expectation (FAO, 2016; Finch-Savage \& Bassel, 2016; Timu et al., 2014; Rodriguez et al., 2015). Mirza (2015) reported that poor quality seed results in skips, excessive thinning or yield reduction diminishing profitability.

Frequent use of seed of unknown quality by agriculture small-scale producers results in the decline in maize productivity. On the other hand, Wambugu et al. (2012) gave an option of the view on farmer saved seed that even though farmer seed seems to have its limitation that causes loss in quality and decrease in yield, it also has its strength such as the presence of local varieties/Landraces that are able to perform well under unfavorable conditions. McGuire (2007) added that farmer seed practices in addition to knowledge and social associations are at the heart of tactics not only for filling the lack of seed but also for handling biotic and abiotic stresses including diseases, pests, and drought. In addition, Seed of open-pollinated variety can be used for more than three years and they retain their genetic characteristics of production (Doss et al., 2003; Spielman et al., 2010). Opposite to the finding of Mueller et al. (2016), Munkvold and White (2016), and Wise et al. (2016) who reported that maize diseases cause important yield loss depending on the disease and the year.

\section{Conclusion}

Seeds from farmer saved and local market are infected with diseases leading to imperfect seedling, susceptible to lodging. Farmer saved seed may constitute the main foundation of dangerous pathogens and may carry unwanted plants which by time could spread and exacerbate the problem in the environment. Though all the seeds sources showed infections of fungal diseases, farmer saved and local market seeds showed high incidence and severity of various diseases compared to the certified seeds from the agro vet. Certainly, certified seed should be of high quality compare to uncertified seed to convince agriculture producers to adopt the improved variety seeds. Seed quality care should be thoroughly controlled and followed up to agro-dealers' stores. Farmers are encouraged to utilize certified seeds to improve crop production, thus to enhance food security.

\section{Acknowledgements}

This publication was made possible through support provided by Alliance for a Green Revolution Africa (AGRA). The opinions expressed herein are those of author(s) and do not necessarily reflect the views of AGRA.

\section{References}

Adebayo, M. A., \& Menkir, A. (2015). Assessment of hybrids of drought-tolerant maize (Zea mays L.) inbred lines for grain yield and other traits under stress managed conditions. Nigerian Journal of Genetics, 28(2), 19-23. https://doi.org/10.1016/j.nigjg.2015.06.004

Adetumbi J. A., \& Olakojo, S. A. (2010). Storage potentials and tolerance of high protein maize (HPM) and quality protein maize (QPM) to seed storage pests in controlled environment. Journal of Plant Protection Research., 50(1), 67-71. https://doi.org/10.2478/v10045-010-0012-6

Alemu, K. (2015). Seed Production and Dissemination Systems Analyses: The Case of Ethiopia. Food Science and Quality Management, 35, 25-38.

Anteneh, M. (2015). TEF (Eragnostis TEF (zucc) Trotter) seed quality variation in East Gojjam zone, Ethiopia. Malaysian Journal of Medical and Biological Research, 2, 54-62.

Atilaw, A. (2010). A baseline survey on the Ethiopian seed sector (p. 116). Submitted to the African Trade Association.

Asea, G., Onaga, G., Phiri, N. A., \& Karanja, D. K. (2010). Quality rice production manual. National Crop Resources Research Institute (NACRRI) \& CABI Africa Publishers, Kampala, Uganda.

Badu-Apraku, B., Fakorede, M. A. B., Lum, A. F., Menkir, A., \& Ouedraogo, M. (2005, May). Demand-driven technologies for sustainable maize production in West and Central Africa. Proceedings of the Fifth Biennial Regional Maize Workshop, IITA-Cotonou, Bénin (pp. 3-6). 
Badu-Apraku, B., Asuboah, R. A., Fakorede, M., \& Asafo-Adjei, B. (2014). Strategies for sustainable maize seed production in West and Central Africa. Journal of New Seeds, 2(3), 85-95.

Bennett, J. W., \& Klich, M. A., (2003). Mycotoxins. Clin. Microbiol. Rev., 16, 497-516. https://doi.org/10.1128/ CMR.16.3.497-516.2003

Bewley, J. D., Bradford, K., \& Hilhorst, H. (2012). Seeds: Physiology of development, germination and dormancy. Springer Science \& Business Media.

Bishaw, Z., Struik, P. C., \& Van Gastel, A. J. G. (2012). Farmers' seed sources and seed quality: 1. Physical and physiological quality. Journal of Crop Improvement, 26(5), 655-692.

Bosque-Perez, N. A., Olojede, S. O., \& Buddenhagen, I. W. (1998). Effect of maize streak virus disease on the growth and yield of maize as influenced by varietal resistance levels and plant stage at time of challenge. Euphytica, 101(3), 307-317. https://doi.org/10.1023/A:1018345921770

Botelho, L. D. S., Zancan, W. L. A., Machado, J. D. C., \& Barrocas, E. N. (2013). Performance of common bean seeds infected by the fungus Sclerotinia sclerotiorum. Journal of Seed Science, 35(2), 153-160. https://doi.org/10.1590/S2317-15372013000200003

Bouis, H. E., \& Welch, R. M. (2010). Biofortification-A sustainable agricultural strategy for reducing micronutrient malnutrition in the global south. Crop Science, 50(Suppl. 1), S-20. https://doi.org/ 10.2135/cropsci2009.09.0531

Bruijin, G. D., Almekinders, C., \& Keune, L. (1994). Farmers' knowledge and practices in smallholder seed production, with special reference to a case study in central America. ILCA/ICARDA Research Planning Workshop on Seed Production by Smallholder Farmers, Addis Ababa (Ethiopia).

CIMMYT. (2004). The CIMMYT Maize Program 2004. Maize Diseases: A Guide for Field Identification (4th ed., pp. 1-119). Mexico, D.F.: International Maize and Wheat Improvement Centre (CIMMYT).

Coutts, B. A., Prince, R. T., \& Jones, R. A. C. (2008). Further studies on Pea seed-borne mosaic virus in cool-season crop legumes: Responses to infection and seed quality defects. Australian Journal of Agricultural Research, 59(12), 1130-1145. https://doi.org/10.1071/AR08113

COMESA (Common Markets for East and Southern Africa). (2014). COMESA seed harmonization implementation plan (p. 98). A COMESA 2014-2020 strategy to implement COMESA seed trade harmonization regulations leading to increased seed production reliability, trade and competitiveness of the seed industry in the COMESA region. COMESA-ACTESA.

CTA. (2014). Seed System, Science and Policy in East and Central Africa.

Delouche, J. C. (1982). Seed quality guidelines for the small farmer. Improved seed for the small farmer (pp. 26-29). Conference proceedings, CIAT (Centro Internacional de Agricultura Tropical), Cali, Colombia.

Desalegn, A. (2017). Review on impact of plant breeding in crop improvement in Ethiopia. International Journal of Research Studies in Agriculture Sciences, 3(9), 26-35.

Du, P. V., Loan, L. C., Cuong, N. D., Nghiep, H. V., \& Thach, N. D. (2001). Survey on seed borne fungi and its effects on grain quality of common rice cultivars in the Mekong Delta. Omonrice, 9, 107-113.

Dornbos, D. L. (1995). Production environment and seed quality. Seed Quality: Basic Mechanisms and Agricultural Implications, 119-152.

Doss, C. R., Mwangi, W. M., Verkuijl, H., \& De Groote, H. (2003). Adoption of maize and wheat technologies in eastern Africa: A synthesis of the findings of 22 case studies.

ECAPAPA (Eastern and Central Africa Programme for Agricultural Policy Analysis). (2004). Harmonization of Seed Policies and Regulations in Eastern Africa (p. 73). Experiences and Lessons Learned. ECAPAPA Monograph Series 6. Eastern and Central Africa Programme for Agricultural Policy Analysis, Entebbe Uganda.

Elmore, R. W., \& Abendroth, L. J. (2006). Multiple ears per node: Iowa. Retrieved from http://www.agronext. iastate.edu/corn/production/management/hybrid/multiple.html

Finch-Savage, W. E. (1995). Influence of seed quality on crop establishment, growth and yield. Seed Quality: Basic Mechanisms and Agricultural Implications, 361-384.

Finch-Savage, W. E., \& Bassel, G. W. (2015). Seed vigour and crop establishment: Extending performance beyond adaptation. Journal of Experimental Botany, 67(3), 567-591. https://doi.org/10.1093/jxb/erv490 
FAOSTAT (Food and Agriculture Organization). (2009). Retrieved from https://www.fao.org

FAO (Food and Agriculture Organization). (2016a). Seed Security Assessment in North Eastern States of Nigeria (p. 64). Nigeria. Rapid SSA NE-Nigeria.

FAO (Food and Agriculture Organization). (2016b). Seed toolkit. Seed quality control and certification. FAO/Africa Seeds Validation workshop. Abidjan, Cote d'Ivoire.

Furtas, R. (2016). Overview of Certified Seed and Farmer Saved Seed. Economics and Competitiveness Branch, Economics Section, Alberta Government, Canada.

Gebremedhin, G. H., Bereket, H., Daniel, B., \& Tesfaye, B. (2015). Effect of biochar on yield and yield components of wheat and post-harvest soil properties in Tigray, Ethiopia. Journal of Fertilizers \& Pesticides, 6(2), 2-5.

Halidu, J., Abubakar, L., Izge, U. A., Ado, S. G., Yakubu, H., \& Haliru, B. S. (2015). Correlation analysis for maize grain yield, other agronomic parameters and Striga affected traits under Striga infested/free environment. Journal of Plant Breeding and Crop Science, 7(1), 9-17. https://doi.org/10.5897/ JPBCS2013.0410

Hipi, A., Surahman, M., \& Ilyas, S. (2013). Seed genetic purity assessment of maize hybrid using microsatellite markers (SSR). International Journal of Applied Science and Technology, 3(5), 66-71.

Huang, J. X., Sui, P., Nie, S. W., Wang, B. B., Nie, Z. J., Gao, W. S., \& Chen, Y. Q. (2011). Effect of maize-legume intercropping on soil nitrate and ammonium accumulation. Journal of Food, Agriculture and Environment, 9(3\&4), 416-419.

IFPRI (International Food Policy Research Institute). (2012). Transforming Agricultural Extension System and Accelerating Agricultural Productivity Growth in the Democratic Republic of Congo. Kinshasa, DR Congo.

IRRI. (2013). Seed quality training manual. Agricultural Engineering Unit, International Rice Research Institute (IRR).

Jaetzold, R., \& Schmidt, H. (1982). Farm handbook of Kenya: National conditions and farm management information of west Kenya. Nyanza and Western Provinces, IIA, 179-222.

Joshi, K. D., Rehman, A. U., Ullah, G., Baloch, A., Hussain, M., Ahmad, J.\& Qamar, M. (2016). Yield and profits from new and old wheat varieties using certified and farmer-saved seeds. Journal of Agricultural Science and Technology B, 6(2016), 141-51. https://doi.org/10.5897/10.17265/2161-6264/2016.03.001

Kariuki, M. T. (2014). Quality status of farm saved bean seed in Maragua sub-county and management of seed-borne disease by seed treatment (MSc. Thesis, University of Nairobi, Kenya).

Khazaei, F., AghaAlikhani, M., Mobasser, S., Mokhtassi-Bidgoli, A., Asharin, H., \& Sadeghi, H. (2016). Evaluation of Wheat (Triticum aestivum L.) Seed Quality of Certified Seed and Farm-Saved Seed in Three Provinces of Iran. Plant Breeding and Seed Science, 73(1), 99-115. https://doi.org/10.1515/plass-2016-0009

Kieran O'Keeffe, (2009). Maize growth and development (pp. 10-12). PROCOP, New Department of Primary Industries, New South Wales.

LVNWSB (Lac Victoria North Water Service Board). (2013). Water point mapping report, Busia County. Netherlands Development Organization (SNV), Kenya.

Louwaars, N., Van Der Burg, W. J., Virk, D. S., \& Witcombe, J. R. (1997). Alternatives for seed regulatory reform: An analysis of variety testing, variety regulation and seed quality control.

Mahender, A., Anandan, A., \& Pradhan, S. K. (2015). Early seedling vigour, an imperative trait for direct-seeded rice: an overview on physio-morphological parameters and molecular markers. Planta, 241(5), 1027-1050. https://doi.org/10.1007/s00425-015-2273-9

Makumbi, D., Kanampiu, F., Mugo, S., \& Karaya, H. (2015). Agronomic Performance and Genotype $\times$ Environment Interaction of Herbicide-Resistant Maize Varieties in Eastern Africa. Crop Science, 55(2), 540-555.

McGuire, S. J. (2007). Vulnerability in farmer seed systems: Farmer practices for coping with seed insecurity for sorghum in Eastern Ethiopia. Economic Botany, 61(3), 211-222. https://doi.org/10.1663/0013-0001(2007) 61[211:VIFSSF]2.0.CO;2

Mew, T. W., \& Gonzales, P. (2002). A handbook of rice seedborne fungi. International Rice Research Institute. 
Mirza, H. (2015). Seed quality handout for student use (p. 17). Sher-e-Bangla Agricultural University.

Mueller, D. S., Wise, K. A., Sisson, A. J., Allen, T. W., Bergstrom, G. C., Bosley, D. B., ... Collins, A. (2016). Corn yield loss estimates due to diseases in the United States and Ontario, Canada from 2012 to 2015. Plant Health Progress, 17(3), 211-222.

Muiru, W. M., Charles, A. K., Kimenju, J. W., Njoroge, K., \& Miano, D. W. (2015). Evaluation of reaction of maize germplasm to common foliar diseases in Kenya. Journal of Natural Sciences Research, 5(1), 140-145.

Munkvold, G. P., \& White, D. G. (2016). Compedium of corn diseases (4th ed., p. 165). American Phytopathological Society Press.

Niaz, I., \& Dawar, S. (2009). Detection of seed borne mycoflora in maize (Zea mays L.). Pak. J. Bot, 4l(1), 443-451.

Nielsen, R. L. (1999). What a Mess! Corny News Network, Purdue Univ. Retrieved from http://www.agry. purdue.edu/ext/corn/news/articles.99/990823b.html

Nielsen, R. L. (2006). A problem with "Bouquets". Purdue University, USA. Retrieved from http://www.kingcorn.org/news/ articles.06/Bouquets-0912.html

Nwanosike, M. R. O., Mabagala, R. B., \& Kusolwa, P. M. (2015). Disease Intensity and Distribution of Exserohilum turcicum Incitant of Northern Leaf Blight of Maize in Tanzania. International Journal of Pure App. Biosci., 3(5), 1-13

Ochichi, P. B. (2015). Effect of cropping systems on the occurrence of fungal and bacterial diseases of legumes in western Kenya (Doctoral dissertation, University of Nairobi, Kenya).

Ogunniyan, D. J., \& Olakojo, S. A. (2014). Genetic variation, heritability, genetic advance and agronomic character association of yellow elite inbred lines of maize (Zea mays L.). Nigerian Journal of Genetics, 28(2), 24-28. https://doi.org/10.1016/j.nigjg.2015.06.005

Oshone, K., Gebeyehu, S., \& Tesfaye, K. (2014). Assessment of common bean (Phaseolus vulgaris L.) Seed quality produced under different cropping systems by smallholder farmers in eastern Ethiopia. African Journal of Food, Agriculture, Nutrition and Development, 14(1), 8566-8584.

Paplomatas, E. J. (2006). Molecular diagnostics of fungal pathogens. Arab Journal of Plant Protection, 24(2), 158-147.

Pathak, N., \& Zaidi, R. K. (2013). Fungi associated with wheat seed discolouration and abnormalities in in-vitro study. Agricultural Sciences, 4(09), 516. https://doi.org/10.4236/as.2013.49069

Peter, T., \& Allen, G. (2015). Abnormal Corn ears. OHIO State University Extension.

Powell, A. A., Matthews, S., \& Oliveira, M. D. A. (1984). Seed quality in grain legumes. Advances in applied Biology.

Qasim, M., \& Hussain, M. (2010). Comparative study of agronomic parameters in synthetic maize varieties. Journal of Agricultural Research, 48(1).

Rajput, M. A., Pathan, M. A., Lodhi, A. M., Shah, G. S., \& Khanzada, K. A. (2005). Studies on seed-borne fungi of wheat in Sindh Province and their effect on seed germination. Pakistan Journal of Botany, 37(1), 181-185.

Rao, R. G. S., Singh, P. M., \& Rai, M. (2006). Storability of onion seeds and effects of packaging and storage conditions on viability and vigour. Scientia Horticulturae, 110(1), 1-6. https://doi.org/10.1016/j.scienta. 2006.06.002

Reid, L. M., Hamilton, R. I., \& Mather, D. E. (1996). Screening maize for resistance to Giberella ear rot. Research Branch, Agriculture and Agri-Food Canada.

Rodríguez, M. V., Barrero, J. M., Corbineau, F., Gubler, F., \& Benech-Arnold, R. L. (2015). Dormancy in cereals (not too much, not so little): About the mechanisms behind this trait. Seed Science Research, 25(2), 99-119. https://doi.org/10.1017/S0960258515000021

Setimela, P. S., \& B Mwangi, W. (2009). Variety testing and release approaches in DTMA project countries in sub-Saharan Africa (p. 44). CIMMYT.

Shekhar, M., \& Kumar, S. (2012). Inoculation methods and disease rating scales for maize diseases. 
Sperling, L. (2001, June). Targeted Seed Aid and Seed System Interventions: Strengthening small farmer seed systems in East and Central Africa. Proceedings of a workshop held in Kampala.

Spielman, D. J., Byerlee, D., Alemu, D., \& Kelemework, D. (2010). Policies to promote cereal intensification in Ethiopia: The search for appropriate public and private roles. Food Policy, 35(3), 185-194. https://doi.org/ 10.1016/j.foodpol.2009.12.002

Stephen, A., Simon, M., \& Kitaka, O. (2011). Busia crop livestock and fisheries assessment for medium and high rainfall areas October, $9^{\text {th }}$ to $20^{\text {th }}$ 2011. Nairobi, Kenya.

Thomison, P. (2007). Tassel Ears in Corn. Ohio State University C.O.R.N. Newsletter 2007-27.

Timu, A. G., Mulwa, R., Okello, J., \& Kamau, M. (2014). The role of varietal attributes on adoption of improved seed varieties: The case of sorghum in Kenya. Agriculture \& Food Security, 3(1), 9. https://doi.org/10.1186/ 2048-7010-3-9

Tonu N. N., Islam M. R., Karim M. M., Chowdhury M. S. M., \& Mia M. A. T., (2017). Quality and health status of farmer saved wheat seed in Bangladesh. International Journal of Research Science \& Management, 4(9), $10-24$.

Tripathi, K. K., Warrier, R., Govila, O. P., \& Ahuja, V. (2011). Biology of Zea mays (Maize) (p. 33). Department of Biotechnology, Ministry of Science \& Technology \& Ministry of Environment and Forests, Government of India.

UPOV. (2009). Maize (Zea mays L.) guidelines for the conduct of tets for Distinctiveness, Uniformity and Stability (p. 62). International Union for the Protection of New Variety of Plants, Geneva.

Wambugu, P. W., Mathenge, P. W., Auma, E. O., \& VanRheenen, H. A. (2012). Constraints to on-farm maize (Zea mays L.) seed production in western Kenya: plant growth and yield.

Wangai, A. W., Redinbaugh, M. G., Kinyua, Z. M., Miano, D. W., Leley, P. K., Kasina, M., ... Jeffers, D. (2012). First report of maize chlorotic mottle virus and maize lethal necrosis in Kenya. Plant Disease, 96(10), 1582-1582. https://doi.org/10.1094/PDIS-06-12-0576-PDN

Welu, G. (2015). Challenges and opportunities of seed multiplication in Eastern Tigray Ethiopia. Journal of Biology, Agriculture and Healthcare, 5(3), 42-53.

Wekesa, E., Mwangi, W., Verkuijl, H., Danda, K., \& De Groote, H. (2003). Adoption of maize production technologies in the coastal lowlands of Kenya. CIMMYT.

Westengen, O. T., Ring, K. H., Berg, P. R., \& Brysting, A. K. (2014). Modern maize varieties going local in the semi-arid zone in Tanzania. BioMed Central Evolutionary Biology, 14(1), 1-12.

Wise, K. A., Mueller, D. S., \& Sisson, A. (2016). A Farmer's guide to corn diseases (p. 173). American Psychopathological Society Press, St Paul, MN.

Wright, M., Donaldson, T., Cromwell, E., \& New, J. (1994). The retention and care of seeds by small-scale farmers (p. 63).

Yadav, V. K., \& Singh, I. S. (2010). Comparative evaluation of maize inbred lines (Zea mays L.) according to DUS testing using morphological, physiological and molecular markers. Agricultural Sciences, 1(03), 131.

Zegeye, F., Alemu, D., Nishikawa, Y., \& Kiyoshi, S. (2014). Perception of farmers and agricultural experts for farmers' saved seed: The case of East Showa, Ethiopia. Sky Journal of Agricultural Research, 3(5), 74-81.

\section{Copyrights}

Copyright for this article is retained by the author(s), with first publication rights granted to the journal.

This is an open-access article distributed under the terms and conditions of the Creative Commons Attribution license (http://creativecommons.org/licenses/by/4.0/). 